\title{
Adenoviral Vector Ad5-CEA(6D) Vaccine
}

National Cancer Institute

\section{Source}

National Cancer Institute. Adenoviral Vector Ad5-CEA(6D) Vaccine. NCI Thesaurus. Code C91373.

A replication-defective, E1- and E2 b-deleted oncolytic adenoviral serotype 5 (Ad5) encoding an epitope of human carcinoembryonic antigen (CEA) with potential antineoplastic activity. Adenoviral vector Ad5-CEA(6D) vaccine expresses a highly immunogenic analogue of CEA [CAP1-(6D)]. Upon administration, this vaccine may induce both humoral and cellular immune responses against tumor cells expressing the CEA antigen, thereby resulting in the immune-mediated inhibition of tumor cell proliferation and tumor cell death. CEA, a tumor-associated antigen, is overexpressed in various tumor cell types. Deletion of early genes E1 and E2 b in Ad5 potentially circumvent preexisting anti-adenovirus immunity and is capable of inducing strong immune responses. 